\title{
X-RAY ASPECTS OF THE IRAS GALAXIES
}

\author{
E.J.A. MEURS \\ Dunsink Observatory \\ Castleknock, Dublin 15, Ireland
}

\begin{abstract}
Several IRAS galaxies have been detected at X-rays, with a variety of satellite observatories. About half of these are classified optically as Seyfert galaxies. Among those not (convincingly) classified as AGN, many have X-ray luminosities for which stellar evolution products offer convenient explanations. Some non-active IRAS galaxies display anomalously high levels of X-ray emission for which several conceivable origins are investigated: optical misclassification, X-ray misidentification, hidden AGN, incidental activity, starburst, environmental sources. X-ray spectral studies and temporal variations constitute important tools for further investigation, for instance to assess the strength of a starburst or to establish signatures of an active core.
\end{abstract}

\section{Introduction}

IRAS galaxies figure regularly in the timelines of the X-ray satellite observatories. Often this refers to active galaxies of some sort and it is not obvious a priori that IRAS galaxies generally would be suitable X-ray targets. In this talk the question is considered why non-active IRAS galaxies could be interesting at X-rays at all. One strong point is that they offer homogeneously selected samples for study. The high levels of starformation experienced by many of these objects constitutes an area of specific interest, which has to be contrasted with the case for hidden active nuclei, given the sometimes strong X-ray emission from these objects. In a wider context, galaxies as considered here have been suggested as significant contributors to the X-ray background. 


\section{Homogeneous samples for study}

The galaxies included in the IRAS Point Source Catalog tend to exhibit increased levels of starformation, as their IR colours demonstrate (Helou 1986). Most of the IRAS galaxies are spiral galaxies and a number of them is classified as active (Seyferts). The IR most luminous cases are referred to as Ultraluminous and a link with QSOs has been suggested (Sanders et al. 1988).

The selection of objects by the IRAS satellite is rather homogeneous and thus provides attractive samples for study, distributed all over the sky. At X-rays, several galaxies from the IRAS Bright Galaxy Survey were studied by David et al. (1992), using data from the Einstein satellite. A larger compilation of Einstein data was published by Green et al. (1992). Based on a multivariate selection of likely extragalactic IRAS sources (Meurs et al. 1988; Boller et al. 1992a), a correlation with ROSAT All Sky Survey data yielded 242 proposed detections out of 14708 target positions (Boller et al. 1992b); optical data on these cases were taken from the NASA Extragalactic Database. Moran et al. (1996) published a dedicated optical follow-up of the majority of objects in this sample.

The Boller et al. results included a dozen of surprisingly X-ray luminous and apparently non-active spiral galaxies, reaching $L_{X}$ values well into the range normally exhibited by proper active galaxies. Initial guesses by Boller et al. suggested active nuclei (possibly hidden), starbursts, or a combination of these two. Subsequent optical spectroscopy has produced contradictory results for the classification of these objects as regards their level of activity ( $c f$. Meurs et al. 1992; Moran et al. 1994). Evidence for non-active galaxies emitting X-rays at such high levels has been put forward by Fruscione and Griffiths (1991), Griffiths et al. (1992, 1995) and Boyle et al. (1995), while a different view was expressed by Moran et al. $(1994,1996)$ and Halpern et al. (1995). Taking the extensive Moran et al. (1996) classification results at face value, trends for decreasing $\mathrm{L}_{X}$ and increasing $\Delta_{o x}$ (optical/X-ray positional difference) are observed when going from Sy1 via Sy2 and Starbursts to normal galaxies (Meurs, to be submitted). This suggests that, from active nuclei towards stellar evolution products, the bodies of the galaxies are increasingly contributing to the observed X-ray emission. This is consistent with ROSAT PSPC results that along the same sequence of objects the X-ray source size increases (Meurs et al., to be submitted).

Archival Rosat PSPC data for 7 X-ray luminous IRAS galaxies have been analysed, concentrating on (1) the exact position of the X-ray source, (2) the X-ray morphology, (3) companion X-ray sources (Meurs and Norci 1996). It turns out that not in all cases the $X$-ray source is consistent with the optical nucleus of the IRAS galaxy. The morphology of the X-ray 
TABLE 1. Results of archival ROSAT PSPC study

\begin{tabular}{l|c|c|c|}
\hline Object & Central & Extended & Companions \\
\hline $01590-3158$ & + & $\square$ & $?$ \\
$10126+7339$ & + & $\square$ & + \\
$10257-4338$ & - & + & - \\
$10303+7401$ & - & - & + \\
$11395+1033$ & p & - & $?$ \\
$13224-3809$ & $?$ & - & $?$ \\
$16155+6831$ & p & + & + \\
\hline
\end{tabular}

sources indicates that some exhibit extended X-ray sources, while several have companion sources close to them. The table shows the results of this (pilot) investigation. Confirmations are indicated by + , rejections by -, weak support by $\square$, uncertain cases by ? in the table. If no improvement of position with astrometry (based on corresponding optical and X-ray point sources) was possible but the X-ray position from the satellite pointing appears consistent with the galaxy nucleus, then this is indicated by $\mathrm{p}$.

The implication of these results is that the evidence is not generally in favour of unrecognized active nuclei, but again that the disk (or perhaps halo) of the galaxy may be responsible for a significant fraction of the X-ray emission. The source extensions and the occurrence of nearby sources may be meaningful in view of the fact that available catalogued optical data in some cases refer to membership of small groups of galaxies.

In view of some ambiguous results from optical spectroscopy, it is interesting to remember that active galaxies might change category due to change of activity class (e.g. NGC4151, Penston and Pérez 1984) or to temporary activity (Rees 1988). Following this suggestion in Meurs and Norci (1996), this very possibility was quoted for the IRAS galaxy NGC5905 (Bade et al. 1996) and also for another case, NGC3256 (Moran et al. 1996).

\section{X-ray diagnostics}

Regarding X-ray luminosities, the apparently non-active IRAS galaxies occupy a position between active and normal galaxies. A particularly interesting question in this connection is how high an X-ray luminosity can be reached by an evolving young stellar population alone (i.e. without invoking an active nucleus). This issue was already considered by David et al (1992) and more progress will be obtained with the help of high-energy 
population synthesis work (e.g. Lipunov et al. 1996; Guillout et al 1996; Norci and Meurs, in preparation).

For individual IRAS galaxies, the current X-ray satellites (ASCA, SAX) provide sufficiently high spectral resolution and wide enough spectral bandwidth that several useful diagnostics can be applied. These are especially interesting for assessing whether active nuclei, hidden at optical wavelengths, could be mainly responsible for the observed X-ray emission. Spectral modelling informs about abundances and physical conditions in starburst components. Hard X-ray tails may be relevant to contributions from nuclear activity, but for completely obscured nuclei scattered Fe $6 \mathrm{keV}$ lines are expected to be seen (e.g. Iwasawa et al. 1993; Ueno et al. 1996; Moran and Lehnert 1997). Cold and possibly also ionized absorbing columns can be measured from the X-ray spectra and refer to conditions near the source. Other X-ray characteristics, like luminosity, morphology and temporal modulation, remain of importance as with previous satellite data.

\section{References}

Bade et al. (1996) AA 309, L35

Boller et al. (1992a) AA 259, 101

Boller et al. (1992b) AA 261, 57

Boyle et al. (1995) MN 272, 462

David et al. (1992) ApJ 388, 82

Fruscione and Griffiths (1991) ApJ 380, L13

Green et al. (1992) MN 254, 30

Griffiths et al. (1992) MN 255, 545

Griffiths et al. (1995) MN 275, 77

Guillout et al. (1996) AA 316, 89

Halpern et al. (1995) ApJ 453, 611

Helou (1986) ApJ 311, L33

Iwasawa et al. (1993) ApJ 409, 155

Lipunov et al. (1996) ApJ 466, 234

Meurs et al. (1988) Astronomy from large databases: scientific objectives and methodological approaches, Murtagh \& Heck (Eds), p. 49

Meurs et al. (1992) Star forming galaxies and their interstellar medium, Franco et al. (Eds), p. 168

Meurs and Norci (1996) Röntgenstrahlung from the Universe, Zimmermann et al. (Eds), MPE Report 263, p. 481

Moran et al. (1994) ApJ 433, L65

Moran et al. (1996) ApJS 106, 341

Moran and Lehnert (1997) ApJ 478, 172

Penston and Pérez (1984) MN 211, P33

Rees (1988) Nature 333, 523

Sanders et al. (1988) ApJ 328, L35

Ueno et al. (1996) PASJ 48, 389 